\title{
Determination of myocardial infarction size in rats by echocardiography and tetrazolium staining: correlation, agreements, and simplifications
}

\author{
L. dos Santos ${ }^{1,2}$, A.F.S. Mello 2 , E.L. Antonio ${ }^{1,2}$ and P.J.F. Tucci ${ }^{1,2}$ \\ ${ }^{1}$ Disciplina de Cardiologia, Departamento de Medicina, ${ }^{2}$ Disciplina de Fisiologia Cardiovascular e \\ Respiratória, Departamento de Fisiologia, Escola Paulista de Medicina, Universidade Federal de São \\ Paulo, São Paulo, SP, Brasil
}

Correspondence to: P.J.F. Tucci, Rua Estado de Israel, 181, Apto. 94, 04022-000 São Paulo, SP, Brasil

Fax: +55-11-5573-7820. E-mail: tucci@fcr.epm.br

\begin{abstract}
Triphenyltetrazolium chloride (TTC) staining and echocardiography $(\mathrm{ECHO})$ are methods used to determine experimental myocardial infarction (MI) size, whose practical applicability should be expanded. Our objectives were to analyze the accuracy of $\mathrm{ECHO}$ in determining infarction size in rats during the first days following coronary occlusion and to test whether a simplified single measurement by TTC correctly indicates MI size, as determined by the average value for multiple slices. Infarction was induced in female Wistar rats by coronary artery occlusion and MI size analysis was performed after the acute (7th day) and chronic periods (after 4 weeks) by ECHO matched with TTC. ECHO and TTC showed similar values of MI size (\% of left ventricle perimeter) in acute (ECHO: $33 \pm 11$, TTC: $35 \pm 14$ ) and chronic (ECHO: $38 \pm 14$, TTC: $39 \pm 13$ periods), and also presented an excellent correlation $(r=0.92, P<0.001)$. Although measurements from different heart planes showed discrepancies, a single measurement acquired from the mid-ventricular level by TTC was a good estimate of Ml size calculated by the average of multiple planes, with minimal disagreement (Bland-Altman test with mean ratio bias of $0.99 \pm 0.07$ ) and close to an ideal correlation $(r=0.99, P<0.001)$. In the present study, ECHO was confirmed as a useful method for the determination of MI size even in the acute phase. Also, the single measure of a mid-ventricular section proposed as a simplification of the TTC method is a satisfactory prediction of average MI extension.
\end{abstract}

Key words: Myocardial infarction size; Echocardiography and triphenyltetrazolium comparison; Bland-Altman test

Research supported by CNPq and FAPESP.

Received July 27, 2007. Accepted December 19, 2007

Coronary artery ligation is often used to produce experimental myocardial infarction (MI) (1-3). Since MI size is a determinant of cardiac remodeling and dysfunction $(4,5)$, establishing the correct quantification of infarction is of critical importance. It has been proven that in vivo echocardiogram $(\mathrm{ECHO})$ satisfactorily predicts infarct size in rats when healing has occurred $(6,7)$; however, no data exist regarding $\mathrm{ECHO}$ accuracy during earlier periods. In addition, histopathology does not present the desirable sensitivity for necrosis during the initial hours after coronary occlusion, while histochemical triphenyltetrazolium chloride (TTC) staining has proven to be useful and appears to be simpler and less expensive $(8,9)$. Accordingly, the objectives of the present study were: 1) to define the accuracy of ECHO in determining $\mathrm{MI}$ size during acute and chronic periods compared to TTC staining, and 2) to determine whether a single measurement of MI size in a mid-ventricular section correctly indicates the infarct extension determined by multiple planes.

All procedures were conducted in agreement with the Guide for the Care and Use of Laboratory Animals $(\mathrm{NIH}$, Pub. No. 85-23, revised 1985) and the Institutional Research Ethics Committee. MI was induced by anterior 


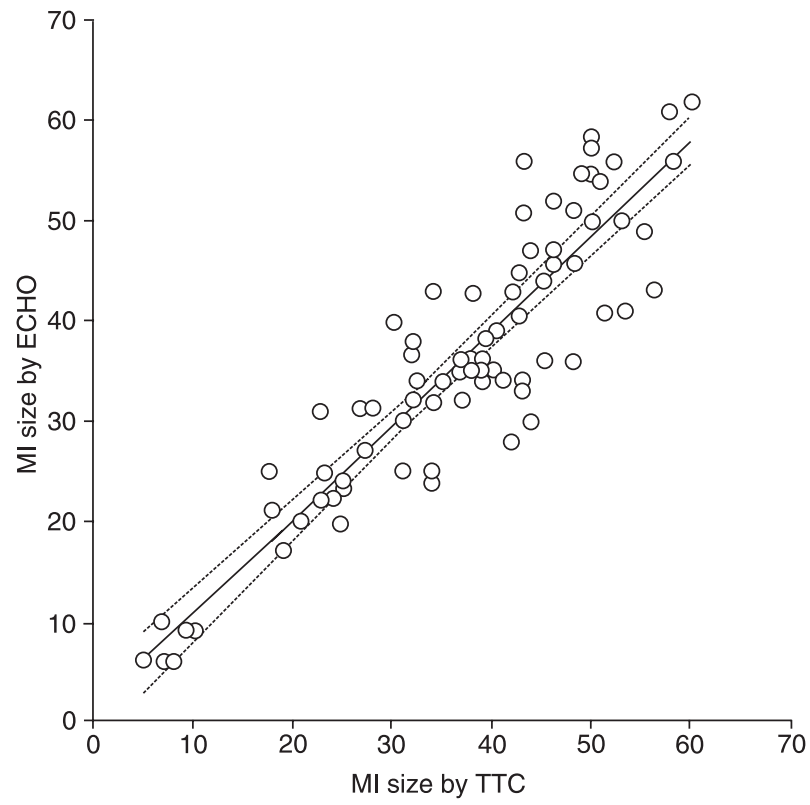

Figure 1. Correlation between $\mathrm{Ml}$ sizes in all infarcted rats performed by matched echocardiography and TTC. The lines represent linear regression plots $(r=0.92, P<0.001)$ and its $95 \%$ confidence interval (dotted lines). ECHO = echocardiography; $\mathrm{MI}$ $=$ myocardial infarction; TTC $=$ triphenyltetrazolium chloride . descending coronary artery ligation $(2,6,7)$ in 116 anesthetized female Wistar rats (180-210 g). The following protocols were performed on the 7th day (acute period, A) and 4 weeks after coronary occlusion (chronic period, C).

Echocardiography was performed as described previously $(6,7)$, with 2-D images from transverse heart planes recorded on 0.5 -inch videotape and off-line analysis of $\mathrm{MI}$ size at basal (tip of the mitral valve leaflets), mid-ventricular (papillary muscle level) and apical (distal to the papillary muscle but beyond the cavity cap) levels. The size of MI was estimated by the percentage of left ventricle (LV) perimeter occupied by the akinetic region, with the arithmetic mean of the three planes determining the final value.

After $\mathrm{ECHO}$, the hearts were excised and sliced from apex to base along the same three transverse planes for MI size evaluation by $1 \%$ TTC according to a previously described technique (8). Pieces were photographed and MI size was calculated (Image Tool 3.0; UTHSCSA, San Antonio, TX, USA) as done for ECHO. In order to test whether a single mid-ventricular section (Single) matched the total Ml size, the section was compared to the final mean value of the measurements obtained from the multiple levels (Multiple).

Data are reported as means \pm SD. Paired Student $t$ -
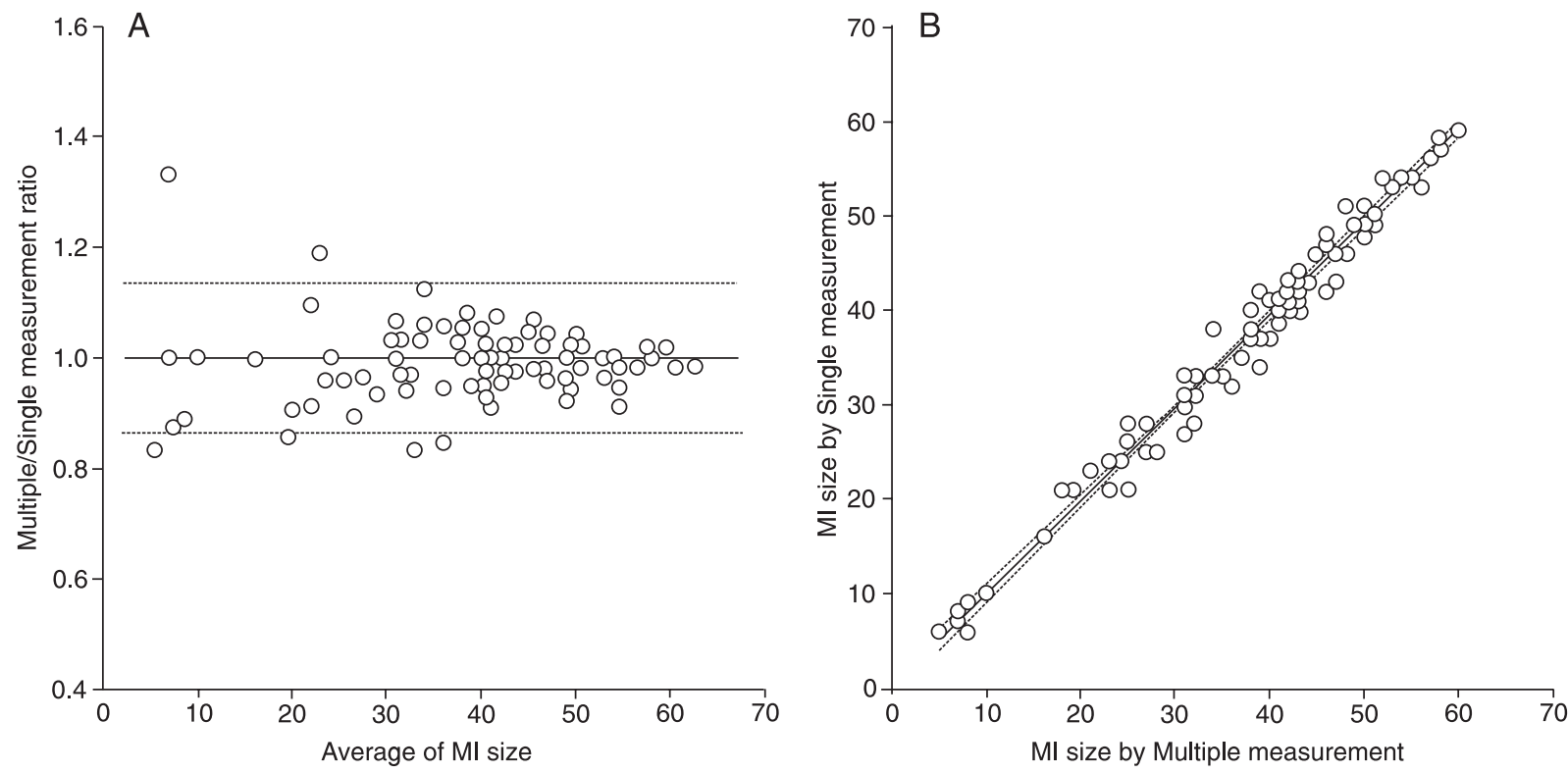

Figure 2. A, Bland-Altman plot of the Multiple/Single measurement ratio as a function of best estimate of the true value (average) in \% of the left ventricle. The continuous line corresponds to the mean bias $(0.99 \pm 0.07)$ and the dotted lines show the $95 \%$ limits of agreement (0.85-1.12). B, Correlation between $\mathrm{MI}$ sizes estimated (in \% of left ventricle) in all rats calculated by Single and Multiple measurements $(r=0.99, \mathrm{P}<0.001)$. MI = myocardial infarction. 
test, Person's correlation, and Bland-Altman analysis were used to evaluate agreement between the measurements. Significance was set at $\mathrm{P}<0.05$.

Analysis of infarctions by ECHO and TTC was performed on 104 surviving rats. Fourteen presented no evidence of an $\mathrm{MI}$ scar and were excluded from the study, leaving 28 infarcted rats in $\mathrm{A}$ and 62 rats in $\mathrm{C}$.

Comparison between ECHO and TTC showed that both methods identified similar values of $\mathrm{MI}$ size (\% of LV) in A (ECHO: $33 \pm 11$, TTC: $35 \pm 14$ ) and C (ECHO: $38 \pm 14$, TTC: $39 \pm 13$ ) and Bland-Altman analysis showed satisfactory agreement between them, resulting in a mean $\mathrm{ECHO} /$ TTC ratio measurement close to the unit in $\mathrm{A}(0.95 \pm 0.1)$ and $C(0.99 \pm 0.14)$. Furthermore, regardless of the coronary occlusion period ( $\mathrm{A}$ and $\mathrm{C}$ in association), comparison also showed a positive correlation between these methods (Figure 1), with a significant correlation coefficient $(r=0.92, P<0.001)$.

As mentioned previously, our other specific interest was to legitimize the Single mid-ventricular measurement by TTC staining in determining $\mathrm{Ml}$ size in comparison to Multiple. The results showed that the ratio between Single and Multiple values presented a mean bias of 0.99 (almost perfect agreement) with minimal deviation (0.07) and a short $95 \%$ confidence interval for discrepancy (0.85-1.12) determined by Bland-Altman analysis (Figure 2A). Single and Multiple values presented an expressive correlation ( $r=0.99, P<0.001$, Pearson) and a slope of 0.98 , characterizing an excellent linear identity (Figure 2B).

Since cardiac remodeling and hemodynamic dysfunc-

\section{References}

1. Johns TN, Olson BJ. Experimental myocardial infarction. I. A method of coronary occlusion in small animals. Ann Surg 1954; 140: 675-682.

2. Bonilha AM, Saraiva RM, Kanashiro RM, Portes LA, Antonio EL, Tucci PJ. A routine electrocardiogram cannot be used to determine the size of myocardial infarction in the rat. Braz J Med Biol Res 2005; 38: 615-619.

3. Spadaro J, Fishbein MC, Hare C, Pfeffer MA, Maroko PR. Characterization of myocardial infarcts in the rat. Arch Pathol Lab Med 1980; 104: 179-183.

4. Pfeffer MA, Pfeffer JM, Fishbein MC, Fletcher PJ, Spadaro $\mathrm{J}$, Kloner RA, et al. Myocardial infarct size and ventricular function in rats. Circ Res 1979; 44: 503-512.

5. Fletcher PJ, Pfeffer JM, Pfeffer MA, Braunwald E. Left ventricular diastolic pressure-volume relations in rats with healed myocardial infarction. Effects on systolic function. Circ Res 1981; 49: 618-626.

6. Cury AF, Bonilha A, Saraiva R, Campos O, Carvalho AC, De Paola AA, et al. Myocardial performance index in female rats with myocardial infarction: relationship with ventricular tion in heart failure after $\mathrm{MI}$ are often proportional to infarct size $(4,5,10)$, a correct interpretation of pathophysiology depends on the satisfactory definition of this variable. Thus, the present finding of adequate agreement between $\mathrm{ECHO}$ and TTC confirms in vivo ultrasound as an acceptable method for determining the extension of lesion, legitimizing its use even in the early phases of MI.

Another important result was the excellent agreement between the Single value of infarct size at the mid-ventricular level and the Multiple measures by TTC, which require laborious, costly and time-consuming manipulation. Consistent with these results, Spadaro et al. (3) showed that the middle transverse plane presents an infarction size close to the mean calculated by planes from the entire ventricle in rats. The Pearson's correlation and the BlandAltman test applied to the data obtained in the present study validate more adequately the Single measurement method rather than the simple comparison of means (3). In the Bland-Altman plot (Figure 2A), some discrepancies can be seen between Single and Multiple measurements for $\mathrm{MI}$ sizes smaller than $25 \%$ of the LV. As these dimensions rarely imply ventricular dysfunction or heart failure (4), these disagreements are less important for pathophysiological studies.

In conclusion, two practical leading concepts resulted from these data: 1) ECHO satisfactorily predicts $\mathrm{MI}$ size in both the acute and chronic phases after coronary occlusion, and 2) a TTC Single measurement from the middle level of the LV convincingly indicates MI size when compared to the mean of Multiple measurements. function parameters by Doppler echocardiography. J Am Soc Echocardiogr 2005; 18: 454-460.

7. Nozawa E, Kanashiro RM, Murad N, Carvalho AC, Cravo $\mathrm{SL}$, Campos O, et al. Performance of two-dimensional Doppler echocardiography for the assessment of infarct size and left ventricular function in rats. Braz $\mathrm{J}$ Med Biol Res 2006; 39: 687-695.

8. Fishbein MC, Meerbaum S, Rit J, Lando U, Kanmatsuse K, Mercier JC, et al. Early phase acute myocardial infarct size quantification: validation of the triphenyl tetrazolium chloride tissue enzyme staining technique. Am Heart J 1981; 101: 593-600.

9. Vivaldi MT, Kloner RA, Schoen FJ. Triphenyltetrazolium staining of irreversible ischemic injury following coronary artery occlusion in rats. Am J Pathol 1985; 121: 522-530.

10. Gschwend S, Buikema H, Henning RH, Pinto YM, de Zeeuw $\mathrm{D}$, van Gilst WH. Endothelial dysfunction and infarct-size relate to impaired EDHF response in rat experimental chronic heart failure. Eur J Heart Fail 2003; 5: 147-154. 